\title{
MRI Findings of Subcutaneous Epidermal Cysts: Emphasis on the Presence of Rupture
}

\author{
Sung Hwan Hong ${ }^{1}$ \\ Hye Won Chung ${ }^{2}$ \\ Ja-Young Choi ${ }^{1}$ \\ Young Hwan Koh ${ }^{1}$ \\ Jung-Ah Choi ${ }^{1}$ \\ Heung Sik Kang ${ }^{1}$
}

Keywords: extremities, MRI, subcutaneous epidermal cyst

DOI:10.2214/AJR.05.0044

Received January 9, 2005; accepted after revision March 2, 2005.

'Department of Radiology, Seoul National University College of Medicine and Institute of Radiation Medicine, 28 Yeongon-dong, Chongno-gu, Seoul, South Korea. Address correspondence to H. S. Kang (kanghs@radcom.snu.ac.kr).

${ }^{2}$ Department of Radiology, Samsung Medical Center, Sungkyunkwan University College of Medicine, 50 Ilwon-dong, Kangnam-gu, Seoul, South Korea.

AJR 2006; 186:961-966

0361-803X/06/1864-961

(C) American Roentgen Ray Society

OBJECTIVE. Our aim was to describe the MRI findings of subcutaneous epidermal cysts with an emphasis on determining the presence of rupture.

CONCLUSION. Epidermal cysts show a fluidlike signal with variable low-signal components on T2-weighted images and peripheral rim enhancement on gadolinium-enhanced images. Most ruptured cysts have septa, show thick and irregular rim enhancement, and are accompanied by a fuzzy enhancement in surrounding subcutaneous tissues. These imaging features of a ruptured epidermal cyst simulate a mass of infectious or neoplastic origin.

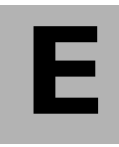
pidermal cysts are cysts filled with keratin debris and bounded by a wall of stratified squamous epithelium [1]. Subcutaneous epidermal cysts commonly involve the scalp, face, neck, trunk, and back; fewer than $10 \%$ occur in the extremities [2]. A few MRI reports have been issued on subcutaneous epidermal cysts [3-7]. Shibata et al. [3] described the MRI features of five cases of epidermal cysts in the extremities. They reported that epidermal cysts have high signal intensity on T2-weighted images and show no enhancement after IV gadolinium administration.

Lee et al. [8] showed the sonographic findings of ruptured epidermal cysts, which can have lobulated contours and show color Doppler signals, mimicking a solid mass. To the best of our knowledge, this is the largest series of MRI cases of subcutaneous epidermal cysts and the second report on the MRI findings of ruptured subcutaneous epidermal cysts after Fisher's case report [9]. The present study was undertaken to determine the MRI features of subcutaneous epidermal cysts, with an emphasis on the presence of rupture.

\section{Materials and Methods}

The records of 24 patients with pathologically confirmed epidermal cysts were retrospectively reviewed. All patients in our study sample were selected from the archives of our institution and were chosen from a review of computer-generated patient diagnoses made between January 1998 and December 2004. The patients included 13 men and 11 women (average age, 42 years). For the limited and anonymous review of patient data required for this study, the institutional review board of our hospital did not require formal approval or informed patient consent.

MRI planes, sequences, and field strengths varied because of the heterogeneous nature of the study group. All patients included in the study had at least one spin-echo T1-weighted MRI and one spin-echo or fast spin-echo T2-weighted MRI. All patients also had IV gadolinium-enhanced MRI studies with or without fat suppression.

MR images were evaluated by consensus between two experienced musculoskeletal radiologists with respect to lesion location, size, shape, and intrinsic characteristics as well as contrast enhancement pattern. Radiologic evaluations included lesion size, shape (round, ovoid, lobulated, or irregular), and border definition (well defined or poorly defined). MRI features of the internal characteristics of epidermal cysts were analyzed in terms of signal intensity, heterogeneity, and septation. Signal intensities of masses were qualitatively compared with those of muscles and fluid. Lesions were assessed on T1-weighted images relative to muscle as having high, low, or mixed signal intensities. On T2-weighted images, signal intensities were classified as high, low, or mixed where the signal intensities of normal muscle were regarded as low, and bright signal intensities of fluid were rated as high.

On gadolinium-enhanced images, we assessed the enhancement patterns of lesions and surrounding soft tissue. We evaluated central and peripheral enhancement in the cyst and focused on the cyst wall shape, that is, thickness and smoothness. The presence or absence of fuzzy enhancement in soft tissue surrounding cysts was also assessed. 


\section{Hong et al.}
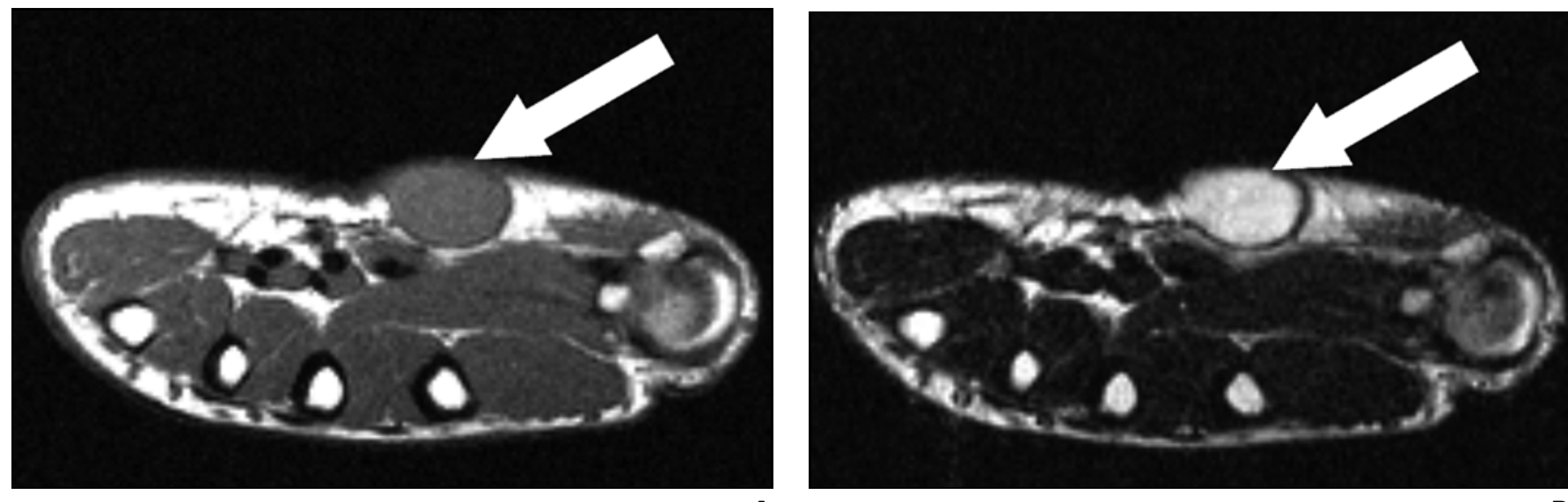

A

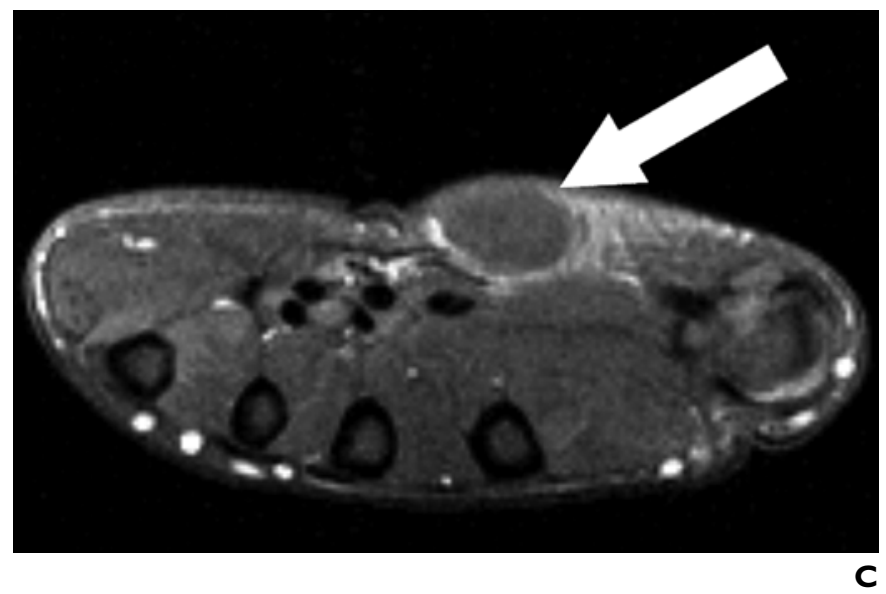

Fig. 1-Unruptured epidermal cyst in subcutaneous layer of left palm in 45-year-old man.

A, Axial T1-weighted image (TR/TE, 600/15) shows well-defined mass (arrow) of slightly high signal intensity.

B, Axial T2-weighted image $(3,500 / 98)$ shows hyperintense mass (arrow).

C, Gadolinium-enhanced fat-suppressed T1-weighted image (600/12) shows mass (arrow) with central nonenhancement and peripheral thin rim enhancement.
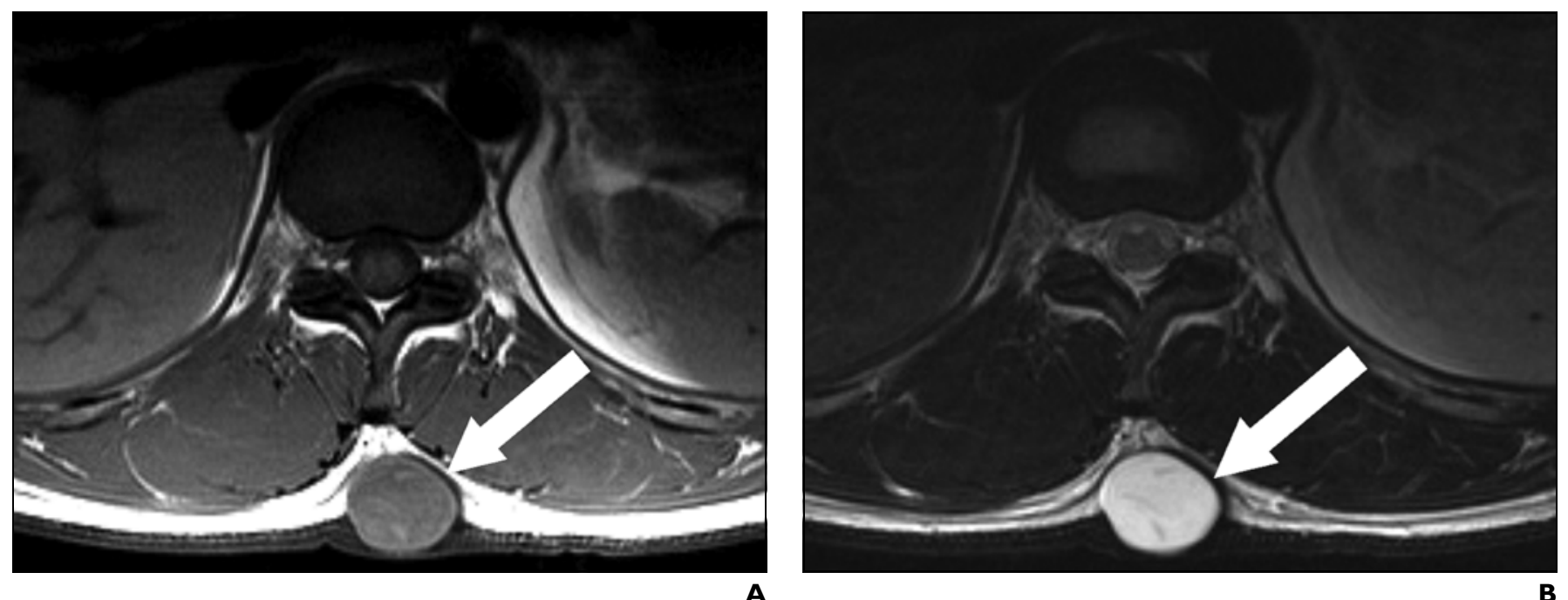

Fig. 2-Unruptured epidermal cyst in back in 26-year-old man

A, Axial T1-weighted image (TR/TE, 638/14) shows round subcutaneous mass (arrow) of slightly high signal intensity. High-signal foci are shown within mass. B, Axial T2-weighted image (3,000/102) shows bright mass (arrow) with nodular low-signal components.

After the surgical excision of epidermal cysts, they were divided into two categories by histopathologic examinations: unruptured or ruptured. We compared the MRI findings of the two groups and then analyzed findings statistically using Fisher's exact test. Significance was accepted at $p<0.01$.

\section{Results}

The locations of the 24 subcutaneous epidermal cysts were as follows: face $(n=1)$, 

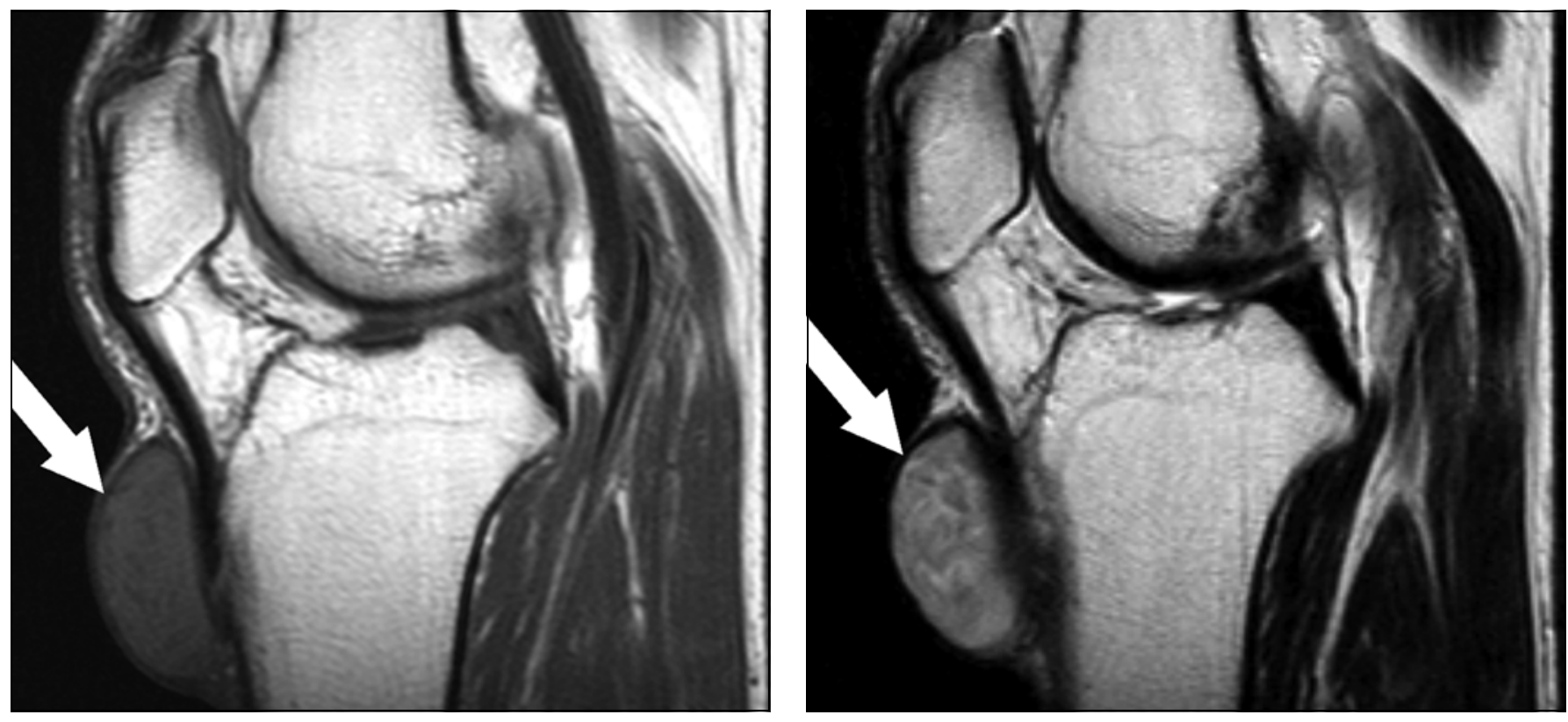

A

Fig. 3-26-year-old man with unruptured epidermal cyst in anterior knee

A, Sagittal T1-weighted image (TR/TE, 500/12) shows ovoid subcutaneous mass (arrow) of slightly high signal intensity.

B, Sagittal T2-weighted image (3,500/105) shows mixed serpentine signal intensity within mass (arrow).
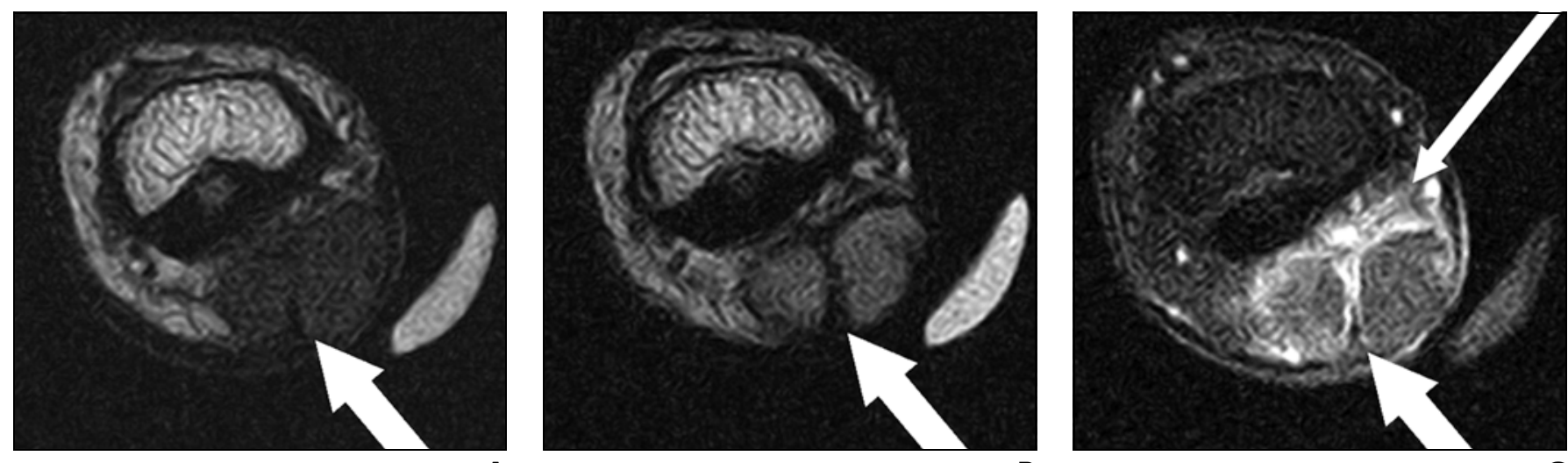

A

B

C

Fig. 4-Ruptured epidermal cyst in right greater toe in 16-year-old girl with recent pain.

A, Axial T1-weighted image (TR/TE, 311/20) shows lobulating subcutaneous mass (arrow) of low signal intensity.

B, Axial T2-weighted image $(1,600 / 100)$ shows hyperintense mass with thick septum (arrow).

C, Gadolinium-enhanced fat-suppressed T1-weighted image (578/20) shows mass with thick irregular peripheral rim enhancement and septal enhancement (thick arrow). Fuzzy soft-tissue enhancement (thin arrow) is also shown around mass.

neck $(n=1)$, shoulder $(n=1)$, back $(n=2)$, coccygeal area $(n=5)$, buttock $(n=3)$, shoul$\operatorname{der}(n=1)$, arm $(n=1)$, hand $(n=2)$, knee $(n=1)$, thigh $(n=2)$, and foot $(n=4)$. Cysts varied in size from 1.2 to $8.1 \mathrm{~cm}$ (mean, 3.5 $\mathrm{cm}$ ), and their shapes also varied, with round $(n=5)$, ovoid $(n=15)$, lobulating $(n=2)$, and irregular $(n=2)$. Twenty-three of 24 cysts had well-defined borders, and one had a poorly defined border.
T2-weighted images showed seven (29\%) lesions of high signal intensity, three (13\%) of low signal intensity, and 14 (58\%) of mixed signal intensity. Lesions of mixed signal intensity had a high background signal with a variable amount of lower signal foci and serpentine structures (Figs. 1-3). On T1weighted images, epidermal cysts revealed low signal intensity in five $(21 \%)$ of 24 , high signal intensity in seven (29\%), and mixed low and high signal intensity in 12 (50\%). Four $(17 \%)$ of the 24 had high signal foci on T1-weighted images (Fig. 2). Ten (42\%) of the 24 lesions had septa (Fig. 4).

On gadolinium-enhanced images, peripheral rim enhancement was seen in all 24 epidermal cysts. We were able to divide rim enhancement patterns into thin and smooth wall enhancement $(n=17,71 \%$ ) (Fig. 1) and thick and irregular wall enhancement ( $n=7,29 \%)$ (Figs. 4 and 5). 


\section{Hong et al.}
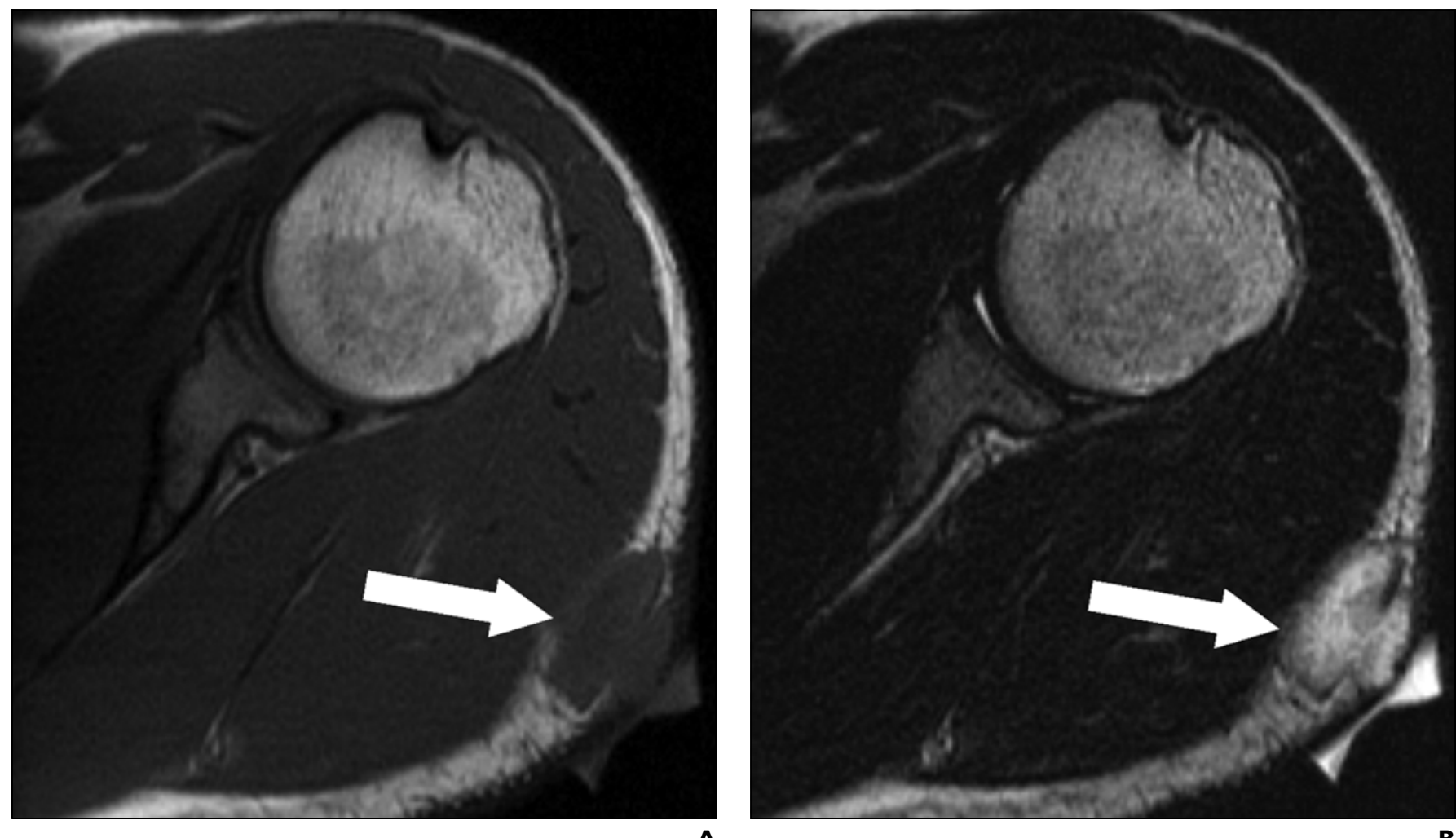

A

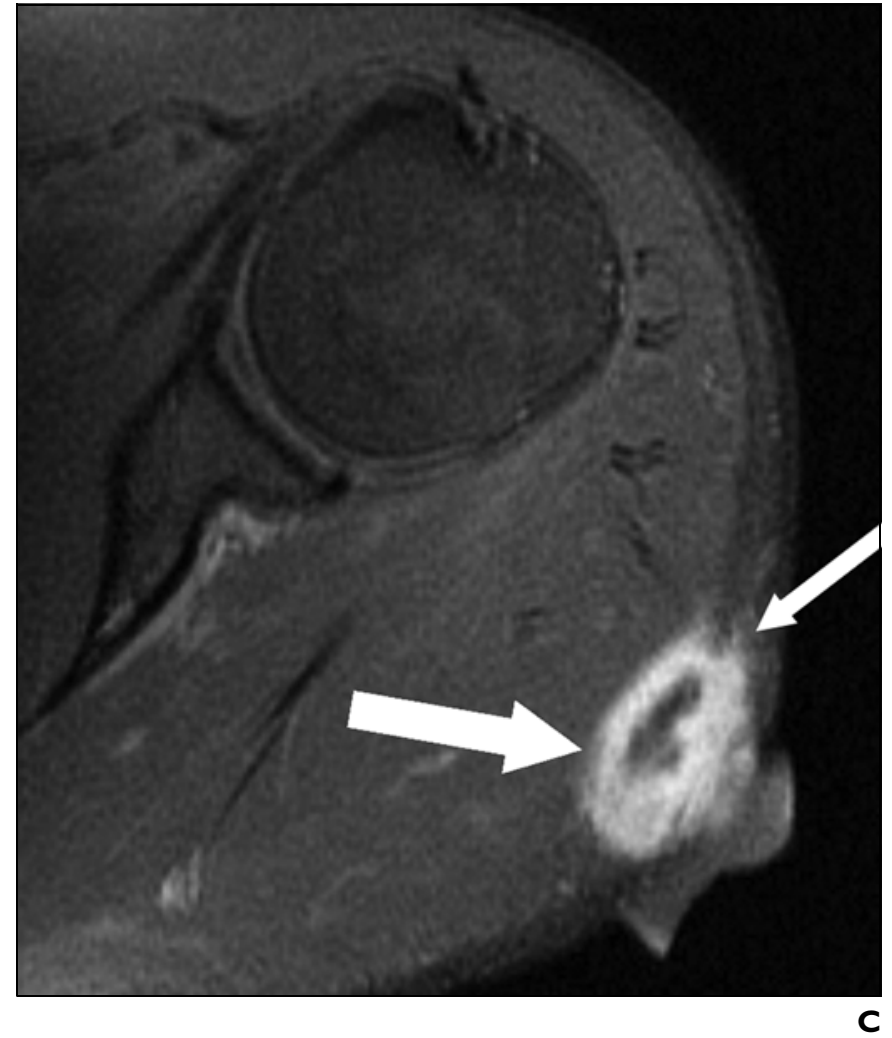

Fig. 5-19-year-old man with painful ruptured epidermal cyst in left posterior shoulder.

A, Axial T1-weighted image (TR/TE, 550/12) shows lobulating subcutaneous mass (arrow) of low signal intensity.

B, Axial T2-weighted image (3,616/108) shows mass (arrow) with central high signal and peripheral low signal intensity.

C, Gadolinium-enhanced fat-suppressed T1-weighted image (516/12) shows mass with very thick and irregular peripheral enhancement (thick arrow). Subtle softtissue enhancement (thin arrow) is present near mass. 


\section{MRI Findings of Subcutaneous Epidermal Cysts}

Histopathologically, the 24 epidermal cysts were classified into two groups: unruptured $(n=16)$ and ruptured $(n=8)$. Table 1 summarizes the differences between the two groups in terms of MRI findings. A significant difference between septation, enhanced rim shapes, and surrounding soft-tissue enhancement was observed between the two cyst types $(p<0.01)$. The majority of ruptured cysts had septa and thick and irregular rim enhancement, which were accompanied by fuzzy enhancement in surrounding subcutaneous tissues.

\section{Discussion}

Epidermal or epidermoid cysts result from the proliferation of epidermal cells within a circumscribed dermal space. Epidermal cysts are probably formed by several mechanismsremnant ectodermal tissues misplaced during embryogenesis [10], occlusion of the pilosebaceous unit, or traumatic or surgical implantation of epithelial elements $[9,11]$. Human papillomavirus type 57 or 60 infections may be additional factors in the development of palmoplantar epidermoid cysts $[12,13]$.

Typically, unruptured epidermal cysts are well-defined round or ovoid lesions of high signal intensity on T2-weighted MR images. In many cases, T2-weighted images show variable low-signal components within epidermal cysts. We speculate that variable signal intensity reflects heterogeneous histopathologic features, as described previously by Brenner et al. [14]. These authors reported the various imaging findings regarding intra- testicular epidermoid cysts, which showed multiple layers of keratin debris, dense debris in cystic centers, and intraluminal calcifications. Moreover, Cho et al. [15] observed high-signal-intensity foci within testicular epidermal cysts on T1-weighted images; however, they did not prove them histopathologically. Fat-suppressed gadolinium-enhanced images were available in two of four cases with these foci; however, they were not suppressed. Gadolinium-enhanced MR images showed central nonenhancement and peripheral thin rim enhancement.

The differential diagnosis of unruptured epidermal cyst includes other subcutaneous cystic masses, some solid tumors, and vascular lesions. Fluid cysts such as ganglion or bursitis also show bright signal intensity on T2-weighted images and peripheral rim enhancement on gadolinium-enhanced images. We believe that the meticulous observation of variable low-signal components in epidermal cysts on T2-weighted images can be used to differentiate epidermal cysts from other fluid cysts. In this study, all unruptured epidermal cysts showed central nonenhancement and peripheral thin rim enhancement without surrounding soft-tissue enhancement. However, using this enhancement pattern alone, we were unable to distinguish them from other cystic masses such as ganglion or bursitis.

Some solid tumors such as neurogenic tumors, nodular fasciitis, myxoid tumors, and dermatofibrosarcoma protuberans may reveal bright signal intensity on T2-weighted images

TABLE I: MRI Findings of Epidermal Cysts

\begin{tabular}{l|c|c}
\hline \multicolumn{1}{c|}{ Imaging Finding } & $\begin{array}{c}\text { Unruptured Cysts } \\
(n=16)\end{array}$ & $\begin{array}{c}\text { Ruptured Cysts } \\
(n=8)\end{array}$ \\
\hline Septation & 3 & 7 \\
Signal intensity (T1-weighted) & 4 & 1 \\
Low & 6 & 1 \\
High & 6 & 6 \\
Mixed & & \\
Signal intensity (T2-weighted) & 1 & 2 \\
Low & 5 & 2 \\
High & 10 & 4 \\
Mixed & & 1 \\
Cyst wall enhancement & 16 & 7 \\
Thin and smooth rim & 0 & 7 \\
Thick and irregular rim & 0 & \\
Soft-tissue enhancement (fuzzy enhancement in surrounding & & \\
subcutaneous tissue) & & \\
\hline
\end{tabular}

and mimic cystic masses [16-19]. However, contrast-enhanced MR images show variable central enhancement within these masses, which is especially helpful for distinguishing solid tumors from epidermal cysts. Hemangioma also presents as a bright mass on T2weighted images and reveals vascular space enhancement after contrast administration.

Ruptured epidermal cysts were not different from unruptured cysts on T1- and T2-weighted images by signal intensity. However, most ruptured epidermal cysts contained septa and showed thick and irregular rim enhancement and fuzzy adjacent soft-tissue enhancement on gadolinium-enhanced images. The peripheral rim and septa were thick and irregular, and their enhancement patterns were reminiscent of inflammatory lesions such as abscesses, complicated ganglion, or bursitis. Clinically, it might be more important to differentiate between ruptured epidermal cysts and other inflammatory or neoplastic lesions than to determine whether an epidermal cyst is ruptured. Epidermal cysts that rupture as a result of trauma can mimic pyogenic collar-button abscesses [11]. Two of eight ruptured epidermal cysts had very thick walls and septa and resembled some malignant soft-tissue tumors with central necrosis (Fig. 5).

Malignant degeneration of epidermal cysts is uncommon, but there have been some reports [20, 21]. Squamous cell carcinomas did occur in $72(2.2 \%)$ of 3,300 epidermal cysts examined [20]. Although we did not have a case of malignant transformation of an epidermal cyst, we believe that MRI findings may be similar between squamous cell carcinomas arising from epidermal cysts and ruptured epidermal cysts. We therefore emphasize that all resected specimens should be scrutinized microscopically, especially in cases with imaging features of ruptured epidermal cyst.

The limitations of our study include the relatively small size of the study sample and the inherent limitations of its retrospective nature. Moreover, we did not perform an MRI-pathology correlation study to explain variable signal intensities within epidermal cysts. Because of the heterogeneous nature of the study group, MRI sequences varied as did imaging planes and scan parameters. Although gadolinium-enhanced MR images were available for all patients, some of them were obtained with fat suppression whereas others were not.

Unruptured subcutaneous epidermal cysts were found to show characteristic MRI findings including a well-demarcated mass, a 


\section{Hong et al.}

high-signal-intensity background with variable low-signal components on T2-weighted images, some bright foci on T1-weighted images, and peripheral thin rim enhancement. An epidermal cyst is more likely than another fluid cyst when the T2-weighted image shows variable low-signal components within a cyst.

On MRI, ruptured epidermal cysts show thick and irregular peripheral rim enhancement, surrounding soft-tissue reactions, and variable septa. Our results indicate that a ruptured epidermal cyst may simulate infectious or neoplastic lesions on MRI and should be included in the differential diagnosis list.

\section{References}

1. Bullough P. Orthopedic pathology, 4th ed. New York, NY: Mosby, 2004:445-446

2. Vincent LM, Parker LA, Mittelstaedt CA. Sonographic appearance of an epidermal inclusion cyst. J Ultrasound Med 1985; 4:609-611

3. Shibata T, Hatori M, Satoh T, Ehara S, Kokubun S. Magnetic resonance imaging features of epidermoid cyst in the extremities. Arch Orthop Trauma Surg 2003; 123:239-241

4. Sundaram M, McGuire MH, Herbold DR, Beshany SE, Fletcher JW. High signal intensity soft tissue masses on T1 weighted pulsing sequences. Skeletal Radiol 1987; 16:30-36
5. Fujimoto H, Murakami K, Kashimada A, et al. Large epidermal cyst involving the ischiorectal fossa: MR demonstration. Clin Imaging 1993; 17:146-148

6. Kransdorf MJ, Jelinek JS, Moser RP, et al. Softtissue masses: diagnosis using MR imaging. AJR 1989; 153:541-547

7. Takano Y, Yokokawa K, Namiki M, Toki K, Okuyama A. Perineal epidermal cyst. Urol Int 1994; 53:53-55

8. Lee HS, Joo KB, Song HT, et al. Relationship between sonographic and pathologic findings in epidermal inclusion cysts. J Clin Ultrasound 2001; 29:374-383

9. Fisher AR, Mason PH, Wagenhals KS. Ruptured plantar epidermal inclusion cyst. AJR 1998; 171:1709-1710

10. McLendon RE. Epidermoid and dermoid tumors. In: Wilkins RH, Rengachary SS, eds. Neurosurgery, 2nd ed. New York, NY: McGraw-Hill, 1996:959-963

11. Ward WA, Labosky DA. Ruptured epidermal inclusion cyst of the palm presenting as collar-button abscess. J Hand Surg [Am] 1985; 10:899-901

12. Lee S, Lee W, Chung S, et al. Detection of human papillomavirus 60 in epidermal cysts of nonpalmoplantar location. Am J Dermatopathol 2003; 25:243-247

13. Kashima M, Takahama H, Baba T, et al. Detection of human papillomavirus type 57 in the tissue of a plantar epidermoid cyst. Dermatology 2003; 207:185-187

14. Brenner JS, Cumming WA, Ros PR. Testicular epidermoid cyst: sonographic and MR findings. (letter) AJR 1989; 152:1344

15. Cho JH, Chang JC, Park BH, Lee JG, Son $\mathrm{CH}$. Sonographic and MR imaging findings of testicular epidermoid cysts. AJR 2002; 178:743-748

16. Murphey MD, Smith WS, Smith SE, Kransdorf MJ, Temple HT. From the archives of the AFIP: imaging of musculoskeletal neurogenic tumors: radiologic-pathologic correlation. RadioGraphics 1999; 19:1253-1280

17. Wang XL, De Schepper AM, Vanhoenacker F, et al. Nodular fasciitis: correlation of MRI findings and histopathology. Skeletal Radiol 2002; 31:155-161

18. Sakaki M, Hirokawa M, Wakatsuki S, et al. Acral myxoinflammatory fibroblastic sarcoma: a report of five cases and review of the literature. Virchows Arch 2003; 442:25-30

19. Torreggiani WC, Al-Ismail K, Munk PL, Nicolaou S, O'Connell JX, Knowling MA. Dermatofibrosarcoma protuberans: MR imaging features. AJR 2002; 178:989-993

20. Bauer BS, Lewis VL Jr. Carcinoma arising in sebaceous and epidermoid cysts. Ann Plast Surg 1980; 5:222-226

21. Lin CY, Jwo SC. Squamous cell carcinoma arising in an epidermal inclusion cyst. Chang Gung Med J 2002; 25:279-282 